\title{
Significance of the Association between Disc Degeneration Changes on Imaging and Low Back Pain: A Review Article
}

\author{
Ahmad Jabir Rahyussalim ${ }^{1}$, Muhammad Luqman Labib Zufar ${ }^{1}$, Tri Kurniawati $^{2}$ \\ ${ }^{1}$ Department of Orthopaedic and Traumatology, Cipto Mangukusumo Hospital, Faculty of Medicine, Universitas Indonesia, Jakarta, Indonesia \\ ${ }^{2}$ Stem Cell and Tissue Engineering Cluster, Cipto Mangukusumo Hospital, MERC Faculty of Medicine, University of Indonesia, Jakarta, Indonesia
}

Low back pain (LBP) is a major health issue resulting in a huge economic burden on the community. It not only increases the medical costs directly, but also raises the disability and loss of productivity in the general population. Symptoms include local pain over the spinal area, pain radiating to the lower leg, stiffness, and muscle tension. LBP is strongly linked with intervertebral disc degeneration that is further associated with the disruption of the complex anatomy of nucleus pulposus, annulus fibrosus, and adjacent supporting structures of the spine. Change in the shape and intensity of nucleus pulposus, decreased disc height, disc herniation, vertebral endplate changes, presence of osteophyte, and posterior high intensity zones are degenerative changes found in imaging studies. Every feature is considered while grading the severity score. Modic changes, DEBIT (disc extension beyond interspace) score, and Pfirrmann criteria are some of the scoring criteria used for evaluating disc degeneration severity. Moreover, the total number and contiguous pattern of affected discs play a crucial role in symptom generation of back pain. Many studies have reported asymptomatic patients. Thus, the correlation between degeneration severity found in imaging study and symptom severity of LBP remain unclear. This review discusses and summarizes the available literature on the significance of the association between the severity of degenerative changes found in imaging study with the presence and intensity of LBP.

Keywords: Low back pain; Intervertebral disc degeneration; Diagnostic imaging

\section{Introduction}

Low back pain (LBP) is the most common symptom associated with musculoskeletal spinal conditions. It can manifest as pain, stiffness, muscle tension between the costal margin and inferior gluteal fold, referred pain from other structures, or even radicular leg pain affecting the lower leg below the knee $[1,2]$. The reported incidence of LBP is $84 \%$ in adults, with an estimated incidence of $18 \%$ in all age groups at any given time [1,3]. Moreover, the probability of experiencing LBP during the lifetime is $80 \%$ [4]. Total $14 \%$ of the patients in the United States (13 million people) who seek medical help and treatment reportedly present with LBP as the chief complaint, with a report of 417 LBP consultations per 10,000 patients, observed mostly in the 45-64-year age group [4-7]. LBP imposes a considerable physical, social, and economic burden on the community. It affects the individuals' functional capacity,

Received Feb 10, 2019; Revised Apr 19, 2019; Accepted May 3, 2019

Corresponding author: Ahmad Jabir Rahyussalim

Department of Orthopaedic and Traumatology, Faculty of Medicine, Universitas Indonesia, Jalan Salemba 6, Kelurahan Kenari, Kecamatan Senen, Jakarta Pusat, DKI Jakarta, 10320, Indonesia

Tel: +62-21-3155996, Fax: +62-21-3929655, E-mail: rahyussalim71@ui.ac.id 
resulting in further disability, decreased productivity, and increased healthcare expenditure. An estimated $\$ 100$ billion expense per year is associated with LBP $[6,8]$.

In patients with LBP, precisely identifying and locating the source of the pain is diagnostically challenging owing to its elusive etiologies. Disc disruption is confirmed to be the most common cause of LBP, with a previous study indicating a strong correlation of disc disruption severity and pain $[9,10]$. Discogenic pain stemming from disc disruption and degeneration further results in pertinent functional spinal motion instability changes owing to muscle spasm or ligament and muscle sprains, anatomical signs of disc prolapse or height loss, mechanical tears or fissures, and biochemical inflammation. All these changes may be associated with various mechanisms via which patients with disc degeneration experience pain $[11,12]$. Imaging techniques, such as plain radiography, computed tomography (CT), or magnetic resonance imaging (MRI) are used in addition to history records and physical examination to diagnose specific changes and sources of pain in patients with LBP. MRI has been the most significant and reliable tool for assessing intervertebral disc pathology, with its signal characteristics reflecting the findings of aging or degeneration [13]. Interpretation and scoring of the structural changes of disc degeneration, narrowing of disc space, endplate changes, disc bulge, facet arthropathy, osteophyte formation, nucleus pulposus (NP), and annulus fibrosus (AF) shape are considered to assess the degree of disc degeneration using MRI. However, deformity, discspace narrowing, and osteophyte formation are common findings on plain radiographs of patients with LBP $[9,13-$ 15].

However, there is lack of clarity regarding the association of such changes with the severity of LBP. Furthermore, the relationship of the degree of degeneration on imaging and severity of pain remains ambiguous. A review comprising 33 studies on $>3,110$ individuals with no history and complaint of back pain showed a high prevalence of spine degeneration [16]. In contrast, several reports have shown that patients with LBP have no marked disc degeneration changes on imaging [17-20]. Another systematic review of five studies has also reported a high correlation of disc degeneration on MRI findings with a high odds ratio (OR) of 2.8 in individuals with LBP [21]. Moreover, surgical intervention based on MRI changes of disc degeneration can prove risky without evidence that surgery can indeed relieve pain. Moreover, obtaining MRI in patients with LBP is associated with extensive morbidities related to behavioral changes, thinking, or psychosocial characteristics of the patients. Positive MRI results increase the patient's stress level, thereby increasing the pain sensitivity and worsening back pain; this places the patient under pressure to seek treatment through consultation, counseling, injections or even surgery to alleviate pain [21-23]. Therefore, the use of MRI is associated with morbidities and consequences.

This review article aimed to evaluate the level of evidence and significance of the relation between degenerative disc (DD) findings on imaging studies and emerging LBP. Moreover, we aimed to evaluate whether the degree of disc degeneration is significantly associated with LBP severity.

\section{Imaging Changes and Severity Grading in Disc Degeneration}

Intervertebral discs are located between the vertebral bodies and are one of the structures constituting the spinal complex anatomy. The vertebral bodies and intervertebral discs are surrounded by joint capsules, ligaments, tendons, and paraspinal muscles with immense innervation to provide structural and functional support. Intervertebral discs consist of inner NP and concentric outer AF. The NP is composed of chondrocytes, a loose network of collagen type II fibers and a matrix of proteoglycan. This composition is responsible for disc hydration. The peripheral AF is composed of densely fibrous collagen type I fibers. Both the structures work in co-ordination to produce a compressible characteristic that can dispense the compressive loads and simultaneously provide a strong cushioning structure that supports the spine [24,25]. Intervertebral disc degeneration owing to aging, microtrauma, nutritional factors, and genetic predisposition results in changes in the common disc characteristics and surrounding structures. Changes in the disc structure and composition result in different intensity and NP shape seen on MRI, typically using sagittal and axial T2-weighted images $[26,27]$. Moreover, decreased disc height, disc herniation, vertebral endplate changes, and posterior high intensity zones (HIZs) are found on routine MRI examination of patients with disc degeneration (Table 1) [14,28].

Benneker et al. [28] in 2005 examined and scored disc findings consisting of signal intensity loss in T2 MRI, NP shape, annular tears, endplate integrity, osteophyte for- 
Table 1. Variables on magnetic resonance imaging finding used in severity scoring system [28]

\begin{tabular}{|c|c|c|c|c|c|c|c|}
\hline Score & $\begin{array}{l}\text { T2-signal } \\
\text { intensity }\end{array}$ & DEBIT & Nucleus shape & Annular tears & $\begin{array}{l}\text { Modic } \\
\text { changes }\end{array}$ & $\begin{array}{l}\text { Endplate } \\
\text { integrity }\end{array}$ & Osteophytes \\
\hline 0 & Normal & Intact & Round/oval & Intact & Normal & Intact & Absent \\
\hline 1 & Intermediate loss & Bulge & Extension into inner annulus & Concentric tears & Type I & Isolated defects & Marginal \\
\hline 2 & Marked loss & Protrusion & Extension into outer annulus & Radial tears & Type II & $\begin{array}{l}\text { Schmorl's node } \\
<5 \mathrm{~mm}\end{array}$ & Discontinuous \\
\hline 3 & Absent signal & $\begin{array}{l}\text { Extrusion/ } \\
\text { sequestration }\end{array}$ & $\begin{array}{l}\text { Extension beyond outer } \\
\text { annulus }\end{array}$ & Transversal tears & Type III & $\begin{array}{l}\text { Schmorl's node } \\
>5 \mathrm{~mm}\end{array}$ & $\begin{array}{c}\text { Continuous, table } \\
\text { osteophyte }\end{array}$ \\
\hline
\end{tabular}

DEBIT, disc extension beyond interspace.

Table 2. Endplate changes using Modic classification [29]

\begin{tabular}{llll} 
Type & T1-weighted images & T2-weighted images & \\
I & Low signal & High signal & Edema and inflammation of bone marrow \\
II & High signal & ISO to high signal & Marrow ischemia; yellow fatty marrow; transformation \\
\hline III & Low signal & Low signal & Sclerosis over subchondral bony area \\
\hline
\end{tabular}

ISO, International Organization for Standardization.

Table 3. Disc degeneration classification using Pfirrmann grading [13]

\begin{tabular}{|c|c|c|c|c|}
\hline Grade & Structure & $\begin{array}{l}\text { Distinction nucleus } \\
\text { and annulus }\end{array}$ & Signal intensity & Height of intervertebral disc \\
\hline I & Homogenous, bright white & Clear & $\begin{array}{l}\text { Hyperintense, isointense to } \\
\text { cerebrospinal fluid }\end{array}$ & Normal \\
\hline ॥ & $\begin{array}{l}\text { Inhomogenous with or without horizontal } \\
\text { bands }\end{array}$ & Clear & $\begin{array}{l}\text { Hyperintense, isointense to } \\
\text { cerebrospinal fluid }\end{array}$ & Normal \\
\hline III & Inhomogenous, gray & Unclear & Intermediate & Normal to slightly decreased \\
\hline IV & Inhomogenous, gray to black & Lost & Intermediate to hypointense & Normal to moderately decreased \\
\hline V & Inhomogenous, black & Lost & Hypointense & Collapsed disc space \\
\hline
\end{tabular}

mation, Modic changes (normal, types 1-3), and DEBIT score (disc extension beyond interspace; intact, bulged, protrusion, extrusion, or sequestration). Each feature was scored from healthy to the most pathologic state, with values ranging from $0-3$. Of the seven features that were examined, T2 signal intensity loss, shape of NP, Modic changes (Table 2) [29], and osteophyte formation were significantly correlated with grading by using the Pfirrmann criteria (Table 3) [13]. Bechara et al. [14] in 2014 used these findings to generate a cumulative MRI score (CMS). Furthermore, studies using the segmentation algorithm calculated the area of the disc and pixel intensities [sum(Int)]. This calculation resulted in MRI index. These subjective and objective parameters were associated with both, the structure and content of the disc [14,28].

A study reported by de Schepper et al. [15] in 2010 used disc-space narrowing and osteophyte presence as individual radiographic features. This study used the previous study as the baseline for grading the severity of radiographic changes in LBP patients. Endplate sclerosis was excluded from the grading because of previous low interclass correlation coefficient for inter-observer reliability. Examination using grading points from 0 to 3 for each feature showed progressing severity in the DD $[15,30,31]$. Other aspects of disc degeneration reported in the severity grading were stenosis, facet joint arthropathy, and neural canal compression. Pfirrmann et al. [13] in 2001 developed a five-grade severity system associated with changes in structural homogeneity, distinction of NP and AF, signal intensity, and IVD height- or disc-space narrowing. This grading system is comprehensive and reliable with adequate intra- and inter-observer reliability 
( $\kappa, 0.69-0.90)$. Changes affecting vertebral body or bone marrow can be classified using Modic changes type 1-3. A combination of Pfirrmann and Modic classification is recommended for use in describing cases of associated bone marrow impairments in patients with disc degeneration $[29,32]$.

\section{Imaging Changes in Asymptomatic Populations with Disc Degeneration}

Disc degeneration is reported to be the most common cause of LBP in the population. Changes in disc degeneration are associated with the aging process, with higher and increased prevalence found in older age groups of the population. The aging process causes aggrecan deprivation and fragmentation, increased amount of keratan sulfate and type 1 collagen in nucleus, with changes in extracellular matrix structure and composition. Moreover, the hydration and structural framework of the disc are distorted and further alter disc behavior and function, making the disc more susceptible to injury. A deranged spinal segment due to disc degeneration causes pain. This is related to increased levels of inflammatory mediators, such as interleukin (IL)-1, tumor necrosis factor (TNF)- $\alpha$, and matrix metalloproteinases (MMPs) over the disruption area and more sensitivity toward any kind of mechanical stress. However, many asymptomatic cases have been reported in patients with observed disc degeneration and facet joint arthritis. Potential underlying mechanisms for this phenomenon are increased supportive action and strength of paraspinal musculature and structures. The muscle will adapt and adjust its twitch time and proprioceptive ability. Other factors, such as better social and psychosocial capability of an individual, help increase the pain threshold as subjective phenomenon [16,33,34].

A study by Brinjikji et al. [16] in 2015 reviewed 33 articles that include 3,110 asymptomatic individuals who had no history of back pain and reported a high prevalence of spine degeneration on imaging findings. Disc degeneration, loss of disc signal intensity, loss of disc height, disc bulging, disc protrusion, annular fissures, facet arthropathy, and spondylolisthesis were observed in the asymptomatic population, with an increased prevalence with age. There was a reported prevalence of $37 \%$ and $96 \%$ for disc degeneration in individuals aged 20 years and 80 years, respectively. Prevalence of signal intensity loss in DD was $17 \%$ in individuals aged 20 years and increased to
$94 \%$ and $97 \%$ in those aged 70 years and 80 years, respectively. A moderate prevalence was found for disc height loss and disc bulge, ranging from $30 \%-50 \%$ in younger individuals. It was estimated that it increased steadily by $1 \%$ annually and resulted in an $84 \%$ prevalence by the age of 80 years. Disc protrusion and annular fissure had an average prevalence throughout all age groups with the highest incidence in the 70 and 80 years age groups. Facet degeneration was not commonly found in the younger population; however, it was prevalent in $69 \%$ of those aged 70 years [16].

Boden et al. [33] in 1990 and Greenberg and Schnell [35] in 1991 have also reported asymptomatic individuals in whom the prevalence of degenerative features increased with age. A study by Jarvik et al. [34,36] performed on initially asymptomatic 148 individuals showed no association of symptom generation with endplate changes, disc degeneration, annular tears, or facet degeneration. However, depression and the psychosocial condition of individuals had a larger hazard ratio $(2.3 ; 95 \%$ confidence interval $[\mathrm{CI}], 1.2-4.4)$ and were suggested to be predictors of LBP prevalence. This study failed to establish an association between degenerative spine changes and LBP and considered the degenerative findings to be part of the normal aging process and not directly related to pain generation and pathological processes. A patient's clinical condition, fitness, daily activities, and psychosocial condition should be considered while interpreting the findings $[29,37]$.

Five features of DD on MRI were reviewed by Ract et al. [24] in 2015, including disc intensity loss, disc height loss, posterior HIZ, disc herniation, and endplate changes. Disc intensity loss or decrease is related to decreased level of proteoglycan that further results in fibrous change of NP and decreases its compressible and elasticity characteristics $[13,24]$. Moreover, cleft formation, disc bulge, and disc collapse can be seen with further progression of decreased proteoglycan production. As stated before, low intensity and decreased disc space or height is commonly found in asymptomatic individuals with an estimated prevalence of $37 \%-96 \%$, depending on age. Mild changes (Pfirrmann grade 3 ) are more commonly observed than moderate to severe changes (Pfirrmann grades 4 and 5), with the prevalence ranging from $26 \%-100 \%$ and $35 \%-$ $72 \%$, respectively. HIZ in the posterior area is related to the hypervascularized granulation tissue $[13,23]$ and is a result of fissuring over the posterior and radial border of 
the disc from NP to AF with L4-L5 and L5-S1 being the common sites of occurrence because of increased compressive stress and pressure of the area. HIZ has a prevalence of $12 \%-56 \%$ in the asymptomatic population and is reported to increase with patient age [26].

Disc herniation, as a feature of degenerative changes, can be further classified into protrusion, extrusion, and sequestration. Moreover, disc herniation is different from disc bulge in that protrusion of $>50 \%$ of the disc circumference results in disc bulge. Protrusion is focal herniation that has a wider base than the body, while extrusion has a narrower base than the body. When there is already continuity deprivation within the disc itself, it is called disc sequestration. The prevalence of disc protrusion is $20 \%-63 \%$ and that of extrusion is $0 \%-24 \%$ in asymptomatic individuals. There are no reported cases of asymptomatic individuals with disc sequestration possibly because of the increased likelihood of nerve compression due to a sequestered disc. Disc herniation can also affect the vertebral body causing intravertebral hernia (Schmorl's node) due to endplate weakness or trauma. Prevalence of this in asymptomatic individuals ranges from 19\%-24\% $[23,26,38,39]$.

Endplate changes are classified using Modic classification types 1-3, with type 3 showing the final stage of endplate condensation. Prevalence of Modic types 1 and 2 in asymptomatic individuals was $0 \%-13 \%$ and $3 \%-25 \%$, respectively. There are no reports of Modic type 3 because it has the most severe biochemical change and disrupted endplate structure near the innervating posterior spinal plexus and its paraspinal innervation $[12,26]$. Having the finding of asymptomatic individuals with high prevalence in all groups of age, disc degeneration and other degenerative changes may be not causally associated with presenting symptoms of LBP. Imaging changes of the disc degenerative process are common in asymptomatic individuals. These findings may be associated with and interpreted as part of the normal aging process instead of a pathologic process. Moreover, structural support from the paraspinal musculature also helps adapt with degenerative changes in the intervertebral disc and decrease the LBP symptoms.

\section{Degenerative Imaging Changes in Population with Low Back Pain}

LBP due to degenerative changes can be linked to many mechanisms, mainly discogenic and facetogenic pain as the two most commonly described forms. Damage to the intervertebral disc is associated with increased inflammatory and damage-associated molecular patterns mediators, such as such as IL-1, IL-6, IL-8, TNF- $\alpha$, and MMPs; therefore, any kind of mechanical stress and strain will result in raised response and reaction of these mediators. Nerve ingrowth in the direction of the central part of the aneural disc is also reported to be due to the chemotactic response toward these inflammatory processes. Accumulation of lactic acid in the avascular central zone of the annular structure of the intervertebral disc is eventually associated with neurogenic pain generation and manifests as LBP. Moreover, low threshold mechanoreceptors covering the fibrous capsule of the facet join perform a proprioceptive function. They comprise neuropeptide $C$, substance $\mathrm{P}$, and gene-related peptide and work as sympathetic efferent fibers. The medial branch of the dorsal ramus that emerges from the nerve root at the same level and one level above also innervate each facet joint so that any disruptions over these structures may become potential sources of pain in LBP patients. Moreover, disc herniation, spinal ligaments thickening, and articular processes hypertrophy may be associated with progressive narrowing of the spinal canal and further result in back pain related to the compression of neurovascular structures $[9,16,17,21,40]$.

Chou et al. [21] in 2011 reviewed five studies on patients with chronic LBP (CLBP). Decreased signal intensity, disc protrusion, reduced disc height, posterior HIZ, endplate changes, and Modic changes were evaluated, and the OR was measured to determine whether there was strong correlation with symptom outcome. An OR of 1.8-2.8 was observed in individuals with CLBP with disc degeneration. Highest OR was found in individuals in occupational cohort studies (OR, 2.8; 95\% CI, 1.4-5.5). Individuals with CLBP with black disc had an OR of 2.1 (95\% CI, 1.0-4.9), while those with both, black and gray discs had an OR of 2.1 (95\% CI, 1.3-3.5). The OR increased with age in individuals with CLBP and disc degeneration, ranging from 2.3 in the 20-30-year age group to 3.9 in the $40-50$-year age group [20,21]. Abnormal disc contour, including disc protrusion, extrusion, and sequestration did not show significantly increased odds for CLBP (OR, 1.3; 95\% CI, $0.7-2.2)$ [41]. However, Visuri et al. [41] in 2005 reported increased odds in younger military personnel aged 19-20 years old for CLBP (OR, 3.2; 95\% CI, 1.4-7.4). Other MRI features, including decreased disc height, annular tear, posterior HIZ, and Modic changes had ORs of 2.5, 2.0, 2.5, 
and 4.2, respectively [40].Takatalo et al. [17] in 2011 reported an association between intervertebral disc degeneration and severity of LBP symptoms. Disc degeneration was evaluated using MRI and Pfirrmann classification (grades 1-5). This classification evaluated the changes in the NP and AF structures and showed the degree of disc degeneration severity. Modic changes, radial tears, HIZ lesions, disc herniation, osteophyte presence, and bone sclerosis were also considered while generating the sum of disc degeneration changes. The degree of pain severity was evaluated using prevalence over time, intensity using a 0-10 numerical rating scale, frequency of episodes, consultation by physicians, pain medication use, and functional limitation. This latent class analysis resulted in five classes, showing an increase in pain severity. A higher sum score for DD was associated with the three most severe classes $(p<0.001)$. An OR of $2.77(1.38-5.57)$ was assigned to individuals with grade 4 who had significant symptoms. When Modic changes, spondylolytic defects, radial tears, and herniations were considered in the calculation, the OR decreased to 2.57 (1.44-4.60) [17]. Lim et al. [42] in 2005 revealed that patients experienced severe pain due to more disrupted discs. Lim et al. [42] reported grades 4 and 5 disc degeneration (80\%), HIZ (56\%) and fissured and ruptured discs (94\%) having concordant pain $(p<0.05)$. Cheung et al. [9] in 2009 also reported common DD in 1,043 individuals with positive association between the degenerative score using Schneiderman's classification and LBP. Furthermore, O'Neill et al. [43] in 2008 reported that a combined evaluation of the loss of nuclear signal, disc bulge, loss of disc height, and HIZ grade II resulted in increased specificity of $92.6 \%$ with decreased sensitivity of $54.7 \%$ in the diagnostic performance of MRI parameters for discogenic pain. This study used the same scoring system used by Takatalo et al. [17]. Increased pain severity from severely disrupted discs (grades 4 and 5) is believed to result from extensive innervation of the inner AF or NP and inflammation or irritation of the disrupted area [4446].

Bechara et al. [14] in 2014 investigated the MRI changes in older adults aged $\geq 65$ years who had CLBP. The \%MRI index-calculated as the product of the percentage of disc total area (disc structure changes) and percentage of disc intensity (disc content changes) - was reported to have a high correlation with pain score in the CLBP population. A lower \%MRI index was associated with increased destruction of the disc, while higher \%MRI index showed a more preserved disc. L4-L5 was found to be the most common site (42\%) for the most degenerated disc, while L3-L4 was the least common site (29\%). Highest correlation with pain symptoms was found in the least degenerated disc, while the most negative correlation was found in the second-most degenerated disc with Pearson correlation values of $\rho=0.47$ and -0.48 , respectively-thought to be associated with a more stable and less inflamed condition of severely degenerated disc [14]. The least degenerated disc is believed to undergo more inflammation and unstable phases [47]. Kettler et al. [48] in 2011 also reported increased stability in flexion/extension and lateral bending in more severe disc degeneration showing more stable structural changes and support in the more degenerated disc. Meanwhile, the CMS had no significant value with respect to the LBP symptoms in the population [14].

De Schepper et al. [15] in 2010 performed a study on 2,819 individual, including 499 (17.7\%) with LBP and 420 (14.9\%) with CLBP, who reported disc-space narrowing or disc height loss of grade 1 or more and in grade 2 or more had significant correlation with LBP, especially in men. The ORs for grade 1 and grade 2 were reported to be 1.9 (95\% CI, 1.4-2.8) and 1.6 (95\% CI, 1.1-2.4), respectively. There was an increase from 1.3 to 2.0 in the OR when there was more than grade 1 narrowing in two or more levels. Men showed a high incidence of this condition with an OR of 2.4 (95\% CI, $1.6-3.4)$, while women had an OR of 1.7 (95\% CI, 1.3-2.3). Grade 2 or more osteophyte formation over all the lumbar discs showed no significant association with back pain. The OR in men was 1.0 (95\% CI, 0.7-1.4), while that in women was $1.2(95 \%$ CI, 0.9-1.6). Despite narrowing being significantly associated with men, disc-space narrowing was more commonly present in women (1,048 women and 637 men) [15] and was thought to be associated with bone and disc structural and biochemical condition because of postmenopausal condition in women. However, a greater frequency and severity of osteophyte presence was more commonly found in men, probably because of higher bone mineral density in men $[49,50]$. Disc-space narrowing and osteophyte formation increased with age, consistent with previous reports $[49,50]$.

Beattie et al. [51] in 2000 observed 408 individuals with LBP symptoms, including local segmental back pain, radiating back pain, bilateral segmental back pain, and others types of pain classified as atypical pain. Most subjects 
$(\mathrm{n}=84)$ had unremarkable radiological findings, while disc disruption without nerve or thecal sac compression was observed in 69 individuals (16.9\%). Disc extrusion was also observed in 44 individuals. We found a significant association between severe nerve compression and distal lower extremity radiating pain ( $p=0.005$ and 0.008 , respectively). High specificity of 0.95 was related to disc extrusion in generating radiating pain [51]. Previous studies have also shown a similar relationship between disc extrusion and severe nerve compression associated with radiating pain. Boos et al. [52] in 1995 showed a high prevalence of symptomatic individuals (96\%) with disc herniations, and Vucetic et al. [53] in 1995 reported radiating pain and bilateral back pain as the main predictors of disc herniation severity. Saleem et al. [54] in 2013 also reported disc degeneration changes in 163 individuals with LBP. MRI findings showed that 103 (94.5\%) ( $p=0.08)$, $74(67 \%)(p=0.23)$, and $73(67 \%)(p=0.30)$ individuals had reduced disc space, disc bulge, and disc protrusion, respectively. L4-L5 and L5-S1 levels were the most common sites of disc degeneration in the study, found in 105 (64.4\%) and 76 (46.6\%) individuals, respectively [54]. LBP and sciatica were reportedly aggravated during walking $(50.5 \%)(p=0.02)$ and standing (44\%) $(p=0.56)$, respectively, showing an association with increased compression and vascular flow compromises $[51,55]$.

Shambrook et al. [55] studied 354 individuals with LBP who had already undergone lumbosacral MRI examination. One or more MRI changes were reported in $86.4 \%$ individuals, while $17.8 \%$ had all four signs of MRI changes (HIZ, disc degeneration, disc herniation, and nerve root compression). All the MRI abnormalities evaluated in the study were not associated with the sudden onset of current LBP episode with OR ranging from 0.9-1.2. Disc degeneration was moderately associated with radiating pain (OR, 1.6) and weakness/numbness below the knee (OR, 1.6), while nerve root compression had the strongest correlations with radiating pain and weakness/numbness below the knee (OR, 2.5 and 1.8, respectively). This study implied the importance of psychological risk factors in the absence of a physical pathology [55]. Luoma et al. [56] in 2000 showed an increased risk of LBP associated with all the MRI features of disc degeneration, such as dark NP, posterior bulge, and anterior bulge with adjusted ORs of 2.0, 2.7, and 3.4, respectively. The 12-month LBP prevalence was $74.4 \%$ and that of sciatic pain was $29.9 \%$. This study also observed an association of occupation with an increased risk of developing LBP. Carpenters who perform dynamic physical work, such as material handling, postural load, climbing, walking on rough surfaces with many obstacles, and changing posture, more frequently were reported to have the highest prevalence of LBP [56]. Berg et al. [57] in 2013 reported a study of 170 candidates with disc prosthesis who all had localized DD with chronic non-radicular LBP (mean total MRI score of 4.4, mean disability score of 42.3 , and mean LBP intensity of 69.3). The total MRI score from the combined MRI findings (Modic changes, decreased NP intensity, decreased disc height, and posterior HIZ present) and each single MRI finding was reported to be non-significantly related to disability and LBP intensity $(p=0.06-1.00, r=-0.15-0.11)$ [58].

Moreover, disc degeneration severity evaluation using the sum total of imaging features and DD patterns were believed to affect the severity of LBP symptoms. In patients with multilevel degenerated discs, Cheung et al. [58] observed the clinical relevance between two-disc degeneration patterns, skipped level disc degeneration (SLDD), and contiguous multilevel disc degeneration (CMDD). In a study on 1,457 individuals, CMDD was reported in 1,156 (79.3\%) patients, while SLDD was reported in 301 (20.7\%) individuals. Further grading and evaluation classified SLDD into five types. CMDD was reportedly associated with an increased probability of LBP (at any time; OR, $1.39 ; p=0.047)$ and pain severity (OR, $1.83 ; p=0.003$ ). Moreover, there was significantly greater prevalence of severe LBP with CMDD $(p=0.039)$ than that with SLDD $(p=0.001)$. This correlation was also found in SLDD type $\mathrm{V}$ (the closest in pattern to CMDD) when compared with type I; SLDD type V was the most severe. A significant association was also reported between the total degenerative disc disease (DDD) score and type of SLDD (type I, least severe; type $\mathrm{V}$, most severe) with a mean DDD score ranging from 3.3 (type I) to 7.6 (type V) $(p<0.001)$ [58]. Another cross-sectional study by Millecamps et al. [59] in 2015 using mice also showed a significant correlation between increased cumulation of the disc degeneration severity score and the generated symptoms of decreased tolerance to axial stretching (LBP symptom emerges) and increased severity of motor impairment, thought to be due to increased disc innervation and sensitivity of local inflammatory mediators [60].

Disruption of the normal architecture and function of the intervertebral disc due to degenerative processes needs 
to be considered as the reason for the generation of back pain. With the damaged structure showing decreased disc height or disc-space narrowing, annular tear, posterior HIZ, and fissured or ruptured disc, inflammation process may occur and interrupt with extensive innervation in the inner $\mathrm{AF}$ and the associated proprioceptive fibers in the paraspinal structures. Compression over the neurovascular structure in the spinal canal may also occur because of disc bulging, disc protrusion, disc extrusion, and disc sequestration that may appear after disc disruption in the degenerative process. Moreover, an increased number of affected levels and more contiguous patterns of disc degeneration are associated with a more profound effect on the spinal biomechanic and kinematics that may result in more severe pain symptoms in individuals $[59,60]$. Nevertheless, with several studies reporting degenerative changes in the MRI examination in LBP patients, the high prevalence in asymptomatic individuals also needs to be considered. It is unclear whether these degenerative changes are the actual source of back pain because many previous studies were based on a cross-sectional design.

\section{The Importance of Imaging Changes and Features of Disc Degeneration}

Imaging studies are an important method used for diagnosing disc degeneration in conjunction with history taking and physical examination. It comprises of lumbar radiographs, CT scan, and MRI. Provocative discography is another common imaging study used to diagnose patients with back pain. Spinal radiographs are more commonly used to eliminate other diagnoses related to the disruption of spinal structures rather than directly diagnose disc degeneration. It is used mainly for the evaluation of bony anatomy and alignment. Formation of osteophyte, narrowing of disc space, narrowing of the foramina, and endplate sclerosis may suggest DDD. CT scan is practical for use in evaluating a pars defect, spondylolisthesis, disc protrusion, or even foraminal stenosis with nerve compression. It demonstrates better findings of bony impairments related to DDD than radiography. The direct evaluation of disc structures, neural compromise, Modic changes, and grading of endplates impairment can be assessed using MRI studies. Loss of signal intensity in T2weighted images is associated with dehydration and loss of hydrogen ions in the DD. Moreover, a HIZ may also be found in the disc and be correlated with increased inflam- mation, disc tear, or annular tear. These structural changes and inflammatory processes can also be associated with the generation of back pain symptoms $[24,25,28]$.

A review of articles by Brinjikji et al. [16] in 2015 regarding MRI changes in asymptomatic individuals revealed a high prevalence of asymptomatic individuals $(n=3,110)$ with MRI features. Teraguchi et al. [61] in 2014 also showed a high prevalence of disc features in older adults with $>90 \%$ prevalence of disc degeneration in individuals aged $>50$ years and $70 \%$ prevalence in those $<50$ years old. The higher prevalence in the older age groups indicates that degenerative imaging features are not necessarily associated with the pathologic process; they may be associated with the normal aging process in humans. Several studies have also reported similar results with no strong correlation between the MRI features of disc degeneration and the presence and severity of pain despite the use of highly sensitive MRI technique to detect degenerative changes [34,36]. Jensen et al. [18] reported a $64 \%$ prevalence of asymptomatic population with abnormalities, and $52 \%, 27 \%, 1 \%$, and $19 \%$ prevalence of disc bulge, protrusion, extrusion, and Schmorl's nodes, respectively. Boden et al. [33] showed $28 \%$ prevalence of disc abnormalities in individuals without LBP. Kanayama et al. [62] also reported a $60 \%-80 \%$ prevalence of imaging abnormalities in asymptomatic individuals. A systematic review by Steffens et al. [63] in 2014 failed to show consistent correlation between LBP and Modic changes, disc degeneration, and disc herniation. Posterior HIZ, disc signal intensity changes, and decreased disc height were also not associated with LBP and intensity. Other studies also failed to establish a strong correlation with the prognostic features of degenerative changes found on radiological examination $[22,63]$.

With a high prevalence of degenerative features in asymptomatic individuals, Modic type 1 change showed an acknowledged predictive value for the presence of pain. This change was more commonly found in symptomatic subjects aged $<50$ years $(19 \%-50 \%)[18,24]$. A study on 2,457 symptomatic individuals showed a positive predictive value (PPV) of $81 \%$ and a specificity of $98 \%$ with Modic type 1 change. Its extent and severity also contributed to the prediction of LBP [64]. The extent commonly exceeded $25 \%$ of the affected endplate and transformed into Modic type 2 change. Modic type 1 was related to increased inflammatory process related to pain generation. Moreover, decreased disc height, low intensity, and bulged 
disc remained with unpretentious PPV and specificity value even in the Pfirrmann grades 3 and 4. A positive but non-significant correlation of these findings was reported by Hancock et al. [65].

Regarding disc herniation, the asymptomatic population had small hernias $<5 \mathrm{~mm}$ in size, while more severe disc sequestration was almost nil. This was believed to be related to neurogenic pain associated with nerve compression that commonly manifests as radiating pain toward the leg and a change in sensation. Another mechanism associated with this condition was the presence of more pain sensitive nerve endings surrounding the disc, especially on the posterior and posterolateral sides. It was also associated with the presence of posterior HIZ. However, posterior HIZ possessed uncertain values for sensitivity (range, $27 \%$ to $81 \%$ ), specificity (range, $79 \%$ to $97 \%$ ), and PPV (range, 53\% to 95\%). Without the presence of disc protrusion, its predictive value remained the least uncertain. Mitra et al. [66] and Stadnik et al. [38] also reported the same results without a strong association with LBP.

A systematic review on a population with CLBP reported a significant correlation of disc degeneration features found on MRI examination with LBP symptoms. However, this study eventually concluded that this association possessed insufficient strength and evidence because of the heterogeneity of the articles included in the study. Moreover, the resultant OR of MRI findings with the presence of CLBP had a wide CI [21]. Regarding the calculation of \%MRI index (showing the severity of structural and content changes of the intervertebral disc) having the greatest significant positive correlation in the least degenerated disc $(\rho=0.42)$ and the highest negative correlation significance $(\rho=-0.42)$ in the second-most degenerated disc, Bechara et al. [14] showed that there was no direct link between increased pain severity and increased degree of disc degeneration. Further, more stable condition in the most degenerated disc-postulated by Kirkaldy-Williswas associated with the lowest back pain score.

De Schepper et al. [15] reported a significant correlation between the number of levels of affected discs and pain severity. The study also reported a more significant association with disc-space narrowing rather than presence of osteophytes, possibly due to increased pressure over the facet joints and spinal ligaments. Moreover, the narrowing disc space was also associated with nerve root or spinal cord impingement. Cheung et al. [58] in 2012 also reported on the association with disc degeneration pat- terns. A contiguous multilevel pattern was associated with significantly higher prevalence of LBP (OR, 1.39; 95\% CI, $1.00-1.93 ; p=0.047)$ and pain severity (OR, 1.83; 95\% CI, 1.23-2.73; $p=0.003)$ than the skipped level pattern $[42,59]$. Berg et al. [57] reported no significant correlation between disability and the degree of LBP in any individual or combined with MRI findings even on bivariate correlation $(r$; range, -0.32 to 0.51$)$.

Radiography, CT, and MRI remain the primary radiological examinations performed for patients with any kind of spinal complaints, especially back pain. Moreover, lumbar discography can be used if more precise information of disc pathology is needed. Radiography is the preferred initial study to evaluate and diagnose spinal pathologies. Furthermore, MRI studies will provide high-quality, detailed information about the anatomic structures of the spinal tissues. MRI remains the most sensitive imaging modality to evaluate spinal soft tissues impairment, especially disc degeneration. It has been used extensively in the detection and severity evaluation of disc degeneration based on the disc structure, morphology, and signal intensity. It is also used in severity grading as per the Pfirrmann scoring system and Modic classification [13,29,32]. Degenerative changes reported using MRI are consistent in the presented studies with a higher prevalence associated with increased age. MRI studies also possess the ability to detect early degenerative changes in asymptomatic individuals. However, studies have shown that degenerative findings in MRI, such as Modic changes, disc signal intensity changes, narrowing of disc space, disc herniation, and posterior HIZ, have uncertain sensitivity, specificity, and predictive value for symptoms of back pain. Moreover, we need to remember that MRI is particularly associated with extensive morbidities of increased psychosocial stress and behavioral changes in patients.

\section{Conclusions}

This study reports a high prevalence of asymptomatic individuals with DD features and changes on MRI. We found a weak association between these changes and the presence of LBP. Modic type 1 change, disc extrusion, and disc sequestration were associated with LBP in most subjects. Moreover, this study also describes a significant relationship between a higher number and more contiguous multilevel pattern of the affected discs with more recognized symptoms and severity of LBP. However, all these 
correlations must be appraised in the context of different characteristics, quantity, and quality of the population and the studies. This consequently results in an insignificant correlation between the degenerative changes found on MRI or other imaging studies and the presence and severity of LBP.

With some of the included study populations are volunteers and not evaluated by multiple observers, this study has certain limitations. Selection bias by the volunteers may be linked with unrepresented general population. Intra- and inter-observer agreement was unclear and inconsistent. Moreover, some of the included studies did not have consistent standardization of the nomenclature and stratification of the degree of severity toward degenerative imaging findings and back pain symptoms. This study included trials performed $>25$ years previously and has insufficient-strength-grade-of-evidence studies. Further, greater use of cross-sectional study designs result in the lack of a direct causal between the assessed variables. Nevertheless, this literature review provides functional and practical information and details that explain the clinical significance and correlation between degenerative findings of imaging studies and symptom generation of LBP. This review will be applicable and helpful not only for clinicians, but also for the patients themselves.

Further longitudinal prospective studies with more precise LBP symptoms and pre-established standard criteria for MRI changes are needed to validate the exact association. Furthermore, psychosocial condition, daily activities, occupation, and other confounding factors must also be adjusted in the study to generate a significant causal correlation between MRI or imaging changes and the presence of LBP and its severity.

\section{Conflict of Interest}

No potential conflict of interest relevant to this article was reported.

\section{Acknowledgments}

The research has grant from Ristekdikti Kalbe Science Award (RKSA) 2018.

\section{References}

1. Balague F, Mannion AF, Pellise F, Cedraschi C. Nonspecific low back pain. Lancet 2012;379:482-91.

2. Mostagi FQ, Dias JM, Pereira LM, et al. Pilates versus general exercise effectiveness on pain and functionality in non-specific chronic low back pain subjects. J Bodyw Mov Ther 2015;19:636-45.

3. Andersson GB. Epidemiological features of chronic low-back pain. Lancet 1999;354:581-5.

4. Mooney V. Presidential address. International Society for the Study of the Lumbar Spine. Dallas, 1986. Where is the pain coming from? Spine (Phila $\mathrm{Pa}$ 1976) 1987;12:754-9.

5. Peng BG. Pathophysiology, diagnosis, and treatment of discogenic low back pain. World J Orthop 2013;4:42-52.

6. Dagenais S, Caro J, Haldeman S. A systematic review of low back pain cost of illness studies in the United States and internationally. Spine J 2008;8:8-20.

7. Jordan KP, Kadam UT, Hayward R, Porcheret M, Young C, Croft P. Annual consultation prevalence of regional musculoskeletal problems in primary care: an observational study. BMC Musculoskelet Disord 2010;11:144.

8. Katz JN. Lumbar disc disorders and low-back pain: socioeconomic factors and consequences. J Bone Joint Surg Am 2006;88 Suppl 2:21-4.

9. Cheung KM, Karppinen J, Chan D, et al. Prevalence and pattern of lumbar magnetic resonance imaging changes in a population study of one thousand fortythree individuals. Spine (Phila Pa 1976) 2009;34:93440.

10. DePalma MJ, Ketchum JM, Saullo T. What is the source of chronic low back pain and does age play a role? Pain Med 2011;12:224-33.

11. Crock HV. A reappraisal of intervertebral disc lesions. Med J Aust 1970;1:983-9.

12. Ito K, Creemers L. Mechanisms of intervertebral disk degeneration/injury and pain: a review. Global Spine J 2013;3:145-52.

13. Pfirrmann CW, Metzdorf A, Zanetti M, Hodler J, Boos N. Magnetic resonance classification of lumbar intervertebral disc degeneration. Spine (Phila $\mathrm{Pa}$ 1976) 2001;26:1873-8.

14. Bechara BP, Agarwal V, Boardman J, et al. Correlation of pain with objective quantification of magnetic 
resonance images in older adults with chronic low back pain. Spine (Phila Pa 1976) 2014;39:469-75.

15. De Schepper EI, Damen J, van Meurs JB, et al. The association between lumbar disc degeneration and low back pain: the influence of age, gender, and individual radiographic features. Spine (Phila Pa 1976) 2010;35:531-6.

16. Brinjikji W, Luetmer PH, Comstock B, et al. Systematic literature review of imaging features of spinal degeneration in asymptomatic populations. AJNR Am J Neuroradiol 2015;36:811-6.

17. Takatalo J, Karppinen J, Niinimaki J, et al. Does lumbar disc degeneration on magnetic resonance imaging associate with low back symptom severity in young Finnish adults? Spine (Phila Pa 1976) 2011;36:2180-9.

18. Jensen MC, Brant-Zawadzki MN, Obuchowski N, Modic MT, Malkasian D, Ross JS. Magnetic resonance imaging of the lumbar spine in people without back pain. N Engl J Med 1994;331:69-73.

19. Bendix T, Kjaer P, Korsholm L. Burned-out discs stop hurting: fact or fiction? Spine (Phila Pa 1976) 2008;33:E962-7.

20. Kjaer P, Leboeuf-Yde C, Korsholm L, Sorensen JS, Bendix T. Magnetic resonance imaging and low back pain in adults: a diagnostic imaging study of 40-year-old men and women. Spine (Phila Pa 1976) 2005;30:1173-80.

21. Chou D, Samartzis D, Bellabarba C, et al. Degenerative magnetic resonance imaging changes in patients with chronic low back pain: a systematic review. Spine (Phila Pa 1976) 2011;36(21 Suppl):S43-53.

22. Chou R, Fu R, Carrino JA, Deyo RA. Imaging strategies for low-back pain: systematic review and metaanalysis. Lancet 2009;373:463-72.

23. Fisher ES, Welch HG. Avoiding the unintended consequences of growth in medical care: how might more be worse? JAMA 1999;281:446-53.

24. Ract I, Meadeb JM, Mercy G, Cueff F, Husson JL, Guillin R. A review of the value of MRI signs in low back pain. Diagn Interv Imaging 2015;96:239-49.

25. Roberts S, Evans H, Trivedi J, Menage J. Histology and pathology of the human intervertebral disc. J Bone Joint Surg Am 2006;88 Suppl 2:10-4.

26. Hadjipavlou AG, Tzermiadianos MN, Bogduk N, Zindrick MR. The pathophysiology of disc degeneration: a critical review. J Bone Joint Surg Br
2008;90:1261-70.

27. Pearce RH, Thompson JP, Bebault GM, Flak B. Magnetic resonance imaging reflects the chemical changes of aging degeneration in the human intervertebral disk. J Rheumatol Suppl 1991;27:42-3.

28. Benneker LM, Heini PF, Anderson SE, Alini M, Ito K. Correlation of radiographic and MRI parameters to morphological and biochemical assessment of intervertebral disc degeneration. Eur Spine J 2005;14:2735.

29. Modic MT, Steinberg PM, Ross JS, Masaryk TJ, Carter JR. Degenerative disk disease: assessment of changes in vertebral body marrow with MR imaging. Radiology 1988;166(1 Pt 1):193-9.

30. Lane NE, Nevitt MC, Genant HK, Hochberg MC. Reliability of new indices of radiographic osteoarthritis of the hand and hip and lumbar disc degeneration. J Rheumatol 1993;20:1911-8.

31. Kettler A, Wilke HJ. Review of existing grading systems for cervical or lumbar disc and facet joint degeneration. Eur Spine J 2006;15:705-18.

32. Modic MT, Masaryk TJ, Ross JS, Carter JR. Imaging of degenerative disk disease. Radiology 1988;168:17786.

33. Boden SD, Davis DO, Dina TS, Patronas NJ, Wiesel SW. Abnormal magnetic-resonance scans of the lumbar spine in asymptomatic subjects: a prospective investigation. J Bone Joint Surg Am 1990;72:403-8.

34. Jarvik JG, Hollingworth W, Heagerty PJ, Haynor DR, Boyko EJ, Deyo RA. Three-year incidence of low back pain in an initially asymptomatic cohort: clinical and imaging risk factors. Spine (Phila Pa 1976) 2005;30:1541-8.

35. Greenberg JO, Schnell RG. Magnetic resonance imaging of the lumbar spine in asymptomatic adults: cooperative study: American Society of Neuroimaging. J Neuroimaging 1991;1:2-7.

36. Jarvik JJ, Hollingworth W, Heagerty P, Haynor DR, Deyo RA. The Longitudinal Assessment of Imaging and Disability of the Back (LAIDBack) Study: baseline data. Spine (Phila Pa 1976) 2001;26:1158-66.

37. Sasiadek MJ, Bladowska J. Imaging of degenerative spine disease: the state of the art. Adv Clin Exp Med 2012;21:133-42.

38. Stadnik TW, Lee RR, Coen HL, Neirynck EC, Buisseret TS, Osteaux MJ. Annular tears and disk herniation: prevalence and contrast enhancement on 
MR images in the absence of low back pain or sciatica. Radiology 1998;206:49-55.

39. Fardon DF, Milette PC; Combined Task Forces of the North American Spine Society; American Society of Spine Radiology; American Society of Neuroradiology. Nomenclature and classification of lumbar disc pathology: recommendations of the combined task forces of the North American Spine Society, American Society of Spine Radiology, and American Society of Neuroradiology. Spine (Phila Pa 1976) 2001;26:E93-113.

40. Paajanen H, Erkintalo M, Parkkola R, Salminen J, Kormano M. Age-dependent correlation of low-back pain and lumbar disc regeneration. Arch Orthop Trauma Surg 1997;116:106-7.

41. Visuri T, Ulaska J, Eskelin M, Pulkkinen P. Narrowing of lumbar spinal canal predicts chronic low back pain more accurately than intervertebral disc degeneration: a magnetic resonance imaging study in young Finnish male conscripts. Mil Med 2005;170:926-30.

42. Lim CH, Jee WH, Son BC, Kim DH, Ha KY, Park CK. Discogenic lumbar pain: association with MR imaging and CT discography. Eur J Radiol 2005;54:431-7.

43. O’Neill C, Kurgansky M, Kaiser J, Lau W. Accuracy of MRI for diagnosis of discogenic pain. Pain Physician 2008;11:311-26.

44. Palmgren T, Gronblad M, Virri J, Kaapa E, Karaharju E. An immunohistochemical study of nerve structures in the anulus fibrosus of human normal lumbar intervertebral discs. Spine (Phila Pa 1976) 1999;24:2075-9.

45. Fagan A, Moore R, Vernon Roberts B, Blumbergs P, Fraser R. ISSLS prize winner: the innervation of the intervertebral disc: a quantitative analysis. Spine (Phila Pa 1976) 2003;28:2570-6.

46. Kirkaldy-Willis WH, Farfan HF. Instability of the lumbar spine. Clin Orthop Relat Res 1982;(165):11023.

47. Allegri M, Montella S, Salici F, et al. Mechanisms of low back pain: a guide for diagnosis and therapy: version 2; peer review: 3 approved. F1000Res 2016;5:1530.

48. Kettler A, Rohlmann F, Ring C, Mack C, Wilke HJ. Do early stages of lumbar intervertebral disc degeneration really cause instability?: evaluation of an in vitro database. Eur Spine J 2011;20:578-84.

49. Pye SR, Reid DM, Smith R, et al. Radiographic fea- tures of lumbar disc degeneration and self-reported back pain. J Rheumatol 2004;31:753-8.

50. Hassett G, Hart DJ, Manek NJ, Doyle DV, Spector TD. Risk factors for progression of lumbar spine disc degeneration: the Chingford Study. Arthritis Rheum 2003;48:3112-7.

51. Beattie PF, Meyers SP, Stratford P, Millard RW, Hollenberg GM. Associations between patient report of symptoms and anatomic impairment visible on lumbar magnetic resonance imaging. Spine (Phila $\mathrm{Pa}$ 1976) 2000;25:819-28.

52. Boos N, Rieder R, Schade V, Spratt KF, Semmer N, Aebi M. 1995 Volvo Award in clinical sciences: the diagnostic accuracy of magnetic resonance imaging, work perception, and psychosocial factors in identifying symptomatic disc herniations. Spine (Phila Pa 1976) 1995;20:2613-25.

53. Vucetic N, Maattanen H, Svensson O. Pain and pathology in lumbar disc hernia. Clin Orthop Relat Res 1995;(320):65-72.

54. Saleem S, Aslam HM, Rehmani MA, Raees A, Alvi AA, Ashraf J. Lumbar disc degenerative disease: disc degeneration symptoms and magnetic resonance image findings. Asian Spine J 2013;7:322-34.

55. Shambrook J, McNee P, Harris EC, et al. Clinical presentation of low back pain and association with risk factors according to findings on magnetic resonance imaging. Pain 2011;152:1659-65.

56. Luoma K, Riihimaki H, Luukkonen R, Raininko R, Viikari-Juntura E, Lamminen A. Low back pain in relation to lumbar disc degeneration. Spine (Phila $\mathrm{Pa}$ 1976) 2000;25:487-92.

57. Berg L, Hellum C, Gjertsen O, et al. Do more MRI findings imply worse disability or more intense low back pain?: a cross-sectional study of candidates for lumbar disc prosthesis. Skeletal Radiol 2013;42:1593602.

58. Cheung KM, Samartzis D, Karppinen J, Luk KD. Are "patterns" of lumbar disc degeneration associated with low back pain?: new insights based on skipped level disc pathology. Spine (Phila Pa 1976) 2012;37:E430-8.

59. Millecamps M, Czerminski JT, Mathieu AP, Stone LS. Behavioral signs of axial low back pain and motor impairment correlate with the severity of intervertebral disc degeneration in a mouse model. Spine J 2015;15:2524-37. 
60. Edgar MA. The nerve supply of the lumbar intervertebral disc. J Bone Joint Surg Br 2007;89:1135-9.

61. Teraguchi M, Yoshimura N, Hashizume H, et al. Prevalence and distribution of intervertebral disc degeneration over the entire spine in a populationbased cohort: the Wakayama Spine Study. Osteoarthritis Cartilage 2014;22:104-10.

62. Kanayama M, Togawa D, Takahashi C, Terai T, Hashimoto T. Cross-sectional magnetic resonance imaging study of lumbar disc degeneration in 200 healthy individuals. J Neurosurg Spine 2009;11:5017.

63. Steffens D, Hancock MJ, Maher CG, Williams C, Jensen TS, Latimer J. Does magnetic resonance imaging predict future low back pain?: a systematic review. Eur J Pain 2014;18:755-65.

64. Thompson KJ, Dagher AP, Eckel TS, Clark M, Reinig JW. Modic changes on MR images as studied with provocative diskography: clinical relevance: a retrospective study of 2457 disks. Radiology 2009;250:84955.

65. Hancock MJ, Maher CG, Latimer J, et al. Systematic review of tests to identify the disc, SIJ or facet joint as the source of low back pain. Eur Spine J 2007;16:1539-50.

66. Mitra D, Cassar-Pullicino VN, McCall IW. Longitudinal study of high intensity zones on MR of lumbar intervertebral discs. Clin Radiol 2004;59:1002-8. 